\title{
TUNNELING IN DOUBLE-BARRIER HETEROSTRUCTURES - COMPARISON OF TIME-DEPENDENT AND STATIONARY APPROACH*
}

\author{
P. Bala, R. Ryszewski AND W. Bala
}

Institute of Physics, N. Copernicus University, Grudziądzka 5, 87-100 Toruń, Poland

The tunneling probability through a double-barrier $\mathrm{ZnSe} / \mathrm{ZnTe}$ structure is calculated using both wave-packet time dependent quantum mechanics and stationary planar-wave approximation. The obtained $I-V$ characteristics are compared and very good consistence in resonant peaks width and position is observed. The absolute value of tunneling probability obtained in time-dependent and stationary approach is also similar.

PACS numbers: 73.40.Gk, 03.65.-w

The dynamical aspects of tunneling, although important for the physical understanding, as well as for applications, have remained largely unclear [1]. Most theoretical works concerning resonant tunneling involve time-independent methods such as finding eigenvalues for quantum wells and superlattice structures. The transmission probability for such structures usually is calculated using the plane-wave approximation and gives no information about the time resolution of the tunneling process, although different characteristic times have been proposed to describe the tunneling process and the passage through the barrier [1].

Recently, the time-dependent quantum mechanics of a wave packet has been successfully used to calculate the tunneling probability for a double barrier structure $[2,3]$. In this way the detailed dynamical picture of the tunneling phenomena has been obtained [4].

In this paper a detailed time-dependent description of the tunneling phenomena is compared with results obtained in the planar-wave approximation. The double-barrier resonant tunneling structure is formed by $n$-type ZnSe layer of width $d_{W}$ (quantum well) sandwiched between $p$-type ZnTe layers of width $d_{B}$ (barrier region). The height of the barrier is $V_{0}=2 \mathrm{eV}$. The double-barrier structure is

*This work is supported in part by the Committee for Scientific Research under grant No. 203299101 and P. Bała is recipient of Polish Science Foundation stipend. 
placed between wide $n$-type ZnSe layers (with length greater than $1000 \AA$ ). The system is investigated under constant voltage $V$ applied across the growth direction. Because of the high resistivity of the ZnTe layers, potential changes only in the barrier regions are assumed. The energy diagram of the structure is presented in the inset in Fig. 1.

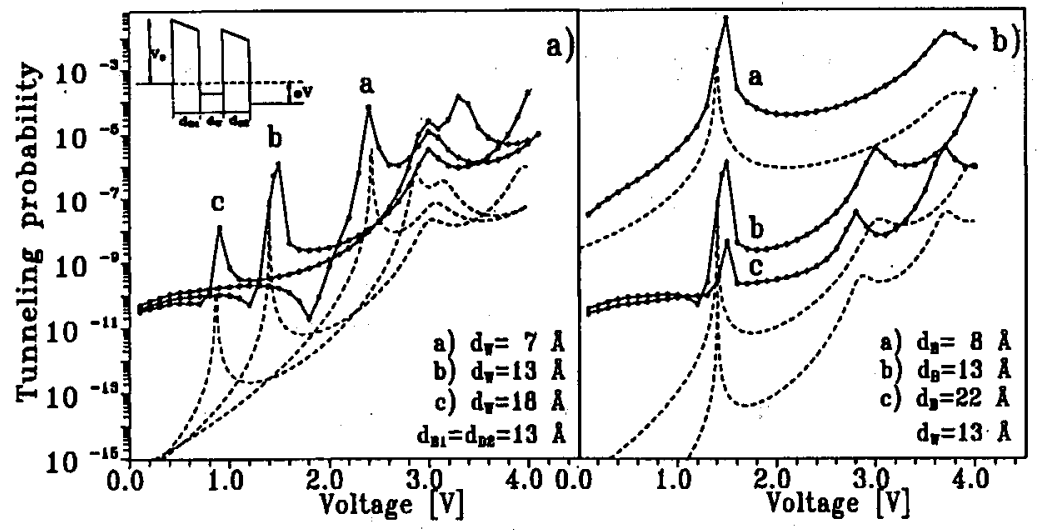

Fig. 1. I- $V$ characteristics for the double-barrier $\mathrm{ZnTe} / \mathrm{ZnSe} / \mathrm{ZnTe}$ structure with different potential well (a) and barrier (b) length. The results for the time-dependent (dashed line) and stationary (solid line) model are presented. The inset presents energy diagram for the double-barrier structure with the applied voltage $V$.

In the time-dependent method (TD) the detailed electron dynamics is obtained by solving, within effective mass approximation [5], the time-dependent Schrödinger equation

$$
\mathrm{i} \hbar \frac{\partial \Psi(x, t)}{\partial t}=\left(-\frac{\hbar^{2}}{2 m^{*}} \frac{\partial^{2}}{\partial x^{2}}+V(x)\right) \Psi(x, t),
$$

where $m^{*}$ is the electron effective mass and depends on the layer type ( $m_{\mathrm{ZnSe}}^{*}=$ $0.16 m_{e}, m_{\mathrm{ZnTe}}^{*}=0.32 m_{e}$ ). The time-dependent Schrödinger equation (1) can be solved using effective and powerful numerical methods as it was reported previously [6] and in a particular case an algorithm based on the Chebychev polynomial expansion [7] has been used.

Within the stationary approach (SA) the wave function is obtained by solving the time-independent Schr "odinger equation

$$
\frac{\partial^{2} \Psi(x)}{\partial x^{2}}+\frac{2 m^{*}}{\hbar^{2}}[E-V(x)] \Psi(x)=0 .
$$

Outside the barrier and in the quantum well region the potential $V(x)$ is constant and the wave function can be approximated by the superposition of incoming and reflected planar waves

$$
\Psi(x)=A \mathrm{e}^{\mathrm{i} k x}+B \mathrm{e}^{-\mathrm{i} k x}, \quad \text { where } \quad k=\sqrt{2 m^{*}(E-V)} .
$$

In the barrier region the potential is a linear function of $x$ and planar wave is no longer a solution of time-independent Schrödinger equation. However, the wave 
function satisfying Eq. (2) can be represented as sum of the Bessel functions $I_{ \pm 1 / 3}$ and $J_{ \pm 1 / 3}[8]$ :

$$
\Psi(x)=\left\{\begin{array}{r}
\sqrt{x_{0}-x}\left[A \left(I_{-1 / 3}(\zeta)-I_{1 / 3}(\zeta)+B\left(I_{-1 / 3}(\zeta)+I_{1 / 3}(\zeta)\right]\right.\right. \\
\text { for } x \leq x_{0} \\
\sqrt{x-x_{0}}\left[A \left(J_{1 / 3}(\zeta)+J_{-1 / 3}(\zeta)+B\left(J_{1 / 3}(\zeta)-J_{-1 / 3}(\zeta)\right]\right.\right. \\
\text { for } \quad x \geq x_{0}
\end{array}\right.
$$

where $\zeta=\frac{2}{3} \sqrt{\frac{m^{*} V}{\hbar^{2} d_{B}}}\left(x-x_{0}\right)^{3 / 2}$ and $x_{0}$ is defined by the equation $E=V\left(x_{0}\right)$. The linear combination coefficients $A$ and $B$ are determined by the continuity condition of the wave function and its first derivative at the edge of each region. The tunneling probability vs. the voltage $V$ applied to the structure, i.e. the $I-V$ characteristics for the different width of the potential well are presented in Fig. 1a. The resonant peaks can be well observed at the same positions for both the time-dependent and the stationary methods. The $I-V$ characteristics for the different barrier width presented in Fig. 1b show the same good agreement in the resonant peak position for both TD and SA methods and the tunneling probability for the resonant conditions is also similar. The remarkable difference is observed in the value of the tunneling probability calculated for nonresonant conditions and the tunneling probability obtained within the SA method is about one order of magnitude smaller than within the TD method. The another important feature of the presented results is a smaller width of the resonant peaks within planar wave approximation. The presented differences can be explained by the fact that within SA model the tunneling probability is calculated according to the incident wave function in planar wave form while the TD calculations were performed with Gaussian-like initial wave packet. The planar wave has exactly determined

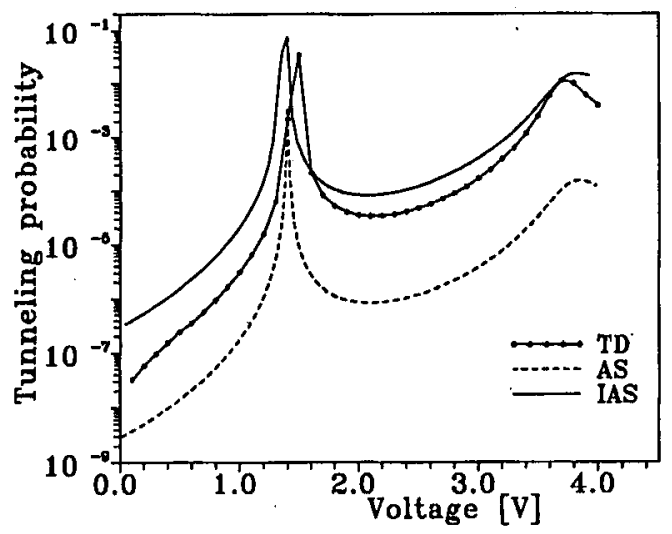

Fig. 2. $I-V$ characteristics for the double-barrier $\mathrm{ZnTe} / \mathrm{ZnSe} / \mathrm{ZnTe}$ structure with dimensions $d_{W}=12 \AA, d_{B 1}=d_{B 2}=13 \AA$. The results for the time-dependent (TD) and stationary (SA) model are presented, as well as planar-wave approximation results integrated over momentum distribution (ISA). 
momentum while Gaussian wave packet is characterized by the momentum distribution of the Gaussian type and can be decomposed to such distribution of the planar waves with different momentum. As it is shown in Fig. 2, tunneling probability within planar-wave approximation integrated over momentum distribution becomes close to time-dependent results and broadening of the resonant peaks is observed.

The presented results show very good consistence in resonant peaks width and position obtained within the stationary and time-dependent theory. The calculated absolute value of tunneling probability is also similar, although the planar-wave approach results must be integrated over momentum distribution. In other case the resonant peak width is underestimated and therefore calculated characteristic times are unreliable.

\section{References}

[1] E.H. Hauge, J.A. Støvneng, Rev. Mod. Phys. 61, 917 (1989).

[2] H. Cruz, A. Munoz, Appl. Phys. A 56, 40 (1993).

[3] P. Bała, J.S. Kwiatkowski, W. Bała, Acta Phys. Pol. A 82, 649 (1992).

[4] P. Bała, W. Bała, Appl. Phys. A, submitted.

[5] R. Tsu, L. Esaki, Appl. Phys. Lett. 2, 562 (1973).

[6] T.N. Truong, J.J. Tanner, P. Bała, J.A. McCammon, D.J. Kouri, B. Lesyng, D.K. Hoffman, J. Chem. Phys. 96, 2077 (1992).

[7] R. Kosloff, H. Tal-Ezer, Chem. Phys. Lett. 127, 223 (1981).

[8] M. Abramowitz, J.A. Stegun, Handbook of Mathematical Functions, Dover 1972. 